Mappemonde

Revue trimestrielle sur l'image géographique et les formes du territoire

$119 \mid 2017$

Varia

\title{
La salinisation des sols dans l'archipel de Kerkennah, Tunisie
}

Lucile Étienne

\section{OpenEdition \\ Journals}

Édition électronique

URL : http://journals.openedition.org/mappemonde/2409

DOI : 10.4000/mappemonde.2409

ISSN : 1769-7298

Éditeur

UMR ESPACE

\section{Référence électronique}

Lucile Étienne, «La salinisation des sols dans l'archipel de Kerkennah, Tunisie », Mappemonde [En ligne], 119 | 2017, mis en ligne le 01 janvier 2017, consulté le 14 septembre 2020. URL : http:// journals.openedition.org/mappemonde/2409

Ce document a été généré automatiquement le 14 septembre 2020.

\section{(c) (i) (2)(2)}

La revue Mappemonde est mise à disposition selon les termes de la Licence Creative Commons Attribution - Pas d'Utilisation Commerciale - Partage dans les Mêmes Conditions 4.0 International. 


\title{
La salinisation des sols dans l'archipel de Kerkennah, Tunisie
}

\author{
Lucile Étienne
}

\author{
Référence de la thèse \\ ÉTIENNE L. (2014). Accentuation récente de la vulnérabilité liée à la mobilité du trait \\ de côte et à la salinisation des sols dans l'archipel de Kerkennah (Tunisie). Thèse \\ de doctorat, Université Paris Diderot (Paris 7), Sorbonne Paris Cité ; Université de \\ Sfax (Faculté des Lettres et Sciences humaines), 2014, $360 \mathrm{p}$.
}

1 L'archipel de Kerkennah est constitué de petites îles et d'îlots dont l'altitude maximale est de seulement 13 mètres. La transition entre la terre et la mer est le plus souvent très douce ce qui implique que de vastes espaces sont touchés par une salinité élevée. Les espaces les plus bas, où la nappe est parfois afFLeurante, sont composés de sebkhas qui sont des terres salées et spécifiques des milieux semi-arides. Dans mon travail, j'ai considéré les sebkhas comme l'ensemble des zones dépourvues et pourvues de végétation halophile caractéristique (chott). À Kerkennah elles représentent $45 \%$ de la surface des îles et sont dites littorales, car elles sont reliées à la mer par un exutoire. Les échanges d'eau entre la terre et la mer sont donc normaux et les sebkhas peuvent être inondées durant la période hivernale, et sèche pendant l'été. Malgré ces espaces très salés, l'agriculture existe sur les îles principales de Gharbi et de Chergui, y compris près de la côte et dans les sebkhas (figure 1). C'est pourquoi une des questions de ma thèse porte sur la salinité des sols agricoles près du littoral et les problématiques qui $\mathrm{y}$ sont associées : quelle est l'extension spatiale de la salinité, quelle est son évolution spatiale et temporelle et comment les agriculteurs réagissent-ils face à elle? 
Figure 1. Occupation du sol en 2010

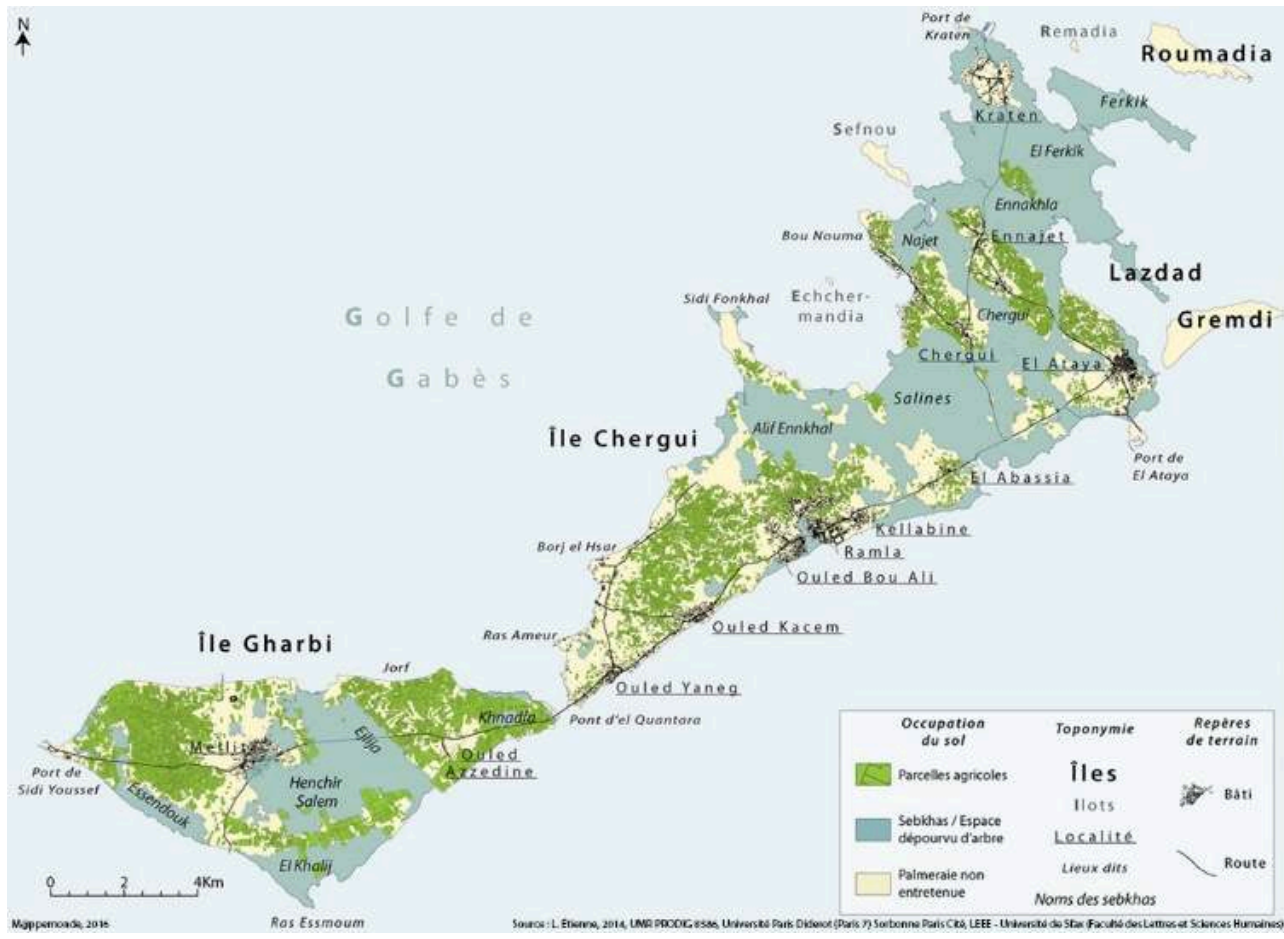

Réalisée à partir d'une image SPOT 5 de 2010, 2,5 m de résolution et de la carte topographique de 1973 au $1: 25000^{e}$

Source : L. Étienne, 2014, UMR PRODIG 8586, Université Paris Diderot (Paris 7) Sorbonne Paris Cité, LEEE - Université de Sfax (Faculté des Lettres et des Sciences Humaines)

Ces questions sont particulièrement importantes en raison de trois facteurs qui touchent l'archipel : (i) l'élévation du niveau de la mer, (ii) l'évolution du climat vers l'aride et (iii) l'évolution des pratiques culturales.

- Le golfe de Gabès connait une élévation du niveau de la mer particulièrement importante au moins depuis les années 1950 (Saidani, 2007), ce qui a pour conséquence le recul de la côte et donc la perte de terres potentiellement arables, mais aussi la salinisation de la nappe souterraine dont l'eau est utilisée pour l'irrigation.

- Le climat évolue vers l'aride depuis les années 1970 avec une élévation des températures et un allongement de la période estivale, ce qui entraine une évaporation et une évapotranspiration plus intenses, et donc un stress hydrique plus important (Dahech, 2012).

L'évolution de ces facteurs naturels implique un stress sur la végétation puisque les plantes ont besoin de plus d'eau et que l'eau disponible est de plus en plus salée. De plus elle a conduit à l'extension parfois importante des sebkhas de l'archipel qui ont, en moyenne, gagné $18 \%$ de leur surface initiale entre 1963 et 2010 (Étienne, 2014).

- Associées aux sècheresses des années 1960 et 1980 (Hénia, in Arnould et Hotyat, 2003), les pratiques culturales ont évolué depuis les années 1960 avec la reconversion de parcelles de palmeraie en oliveraie, jusqu'à l'installation des périmètres irrigués et drainés de Melitta ( figure 2- représenté en vert foncé) et de Ramla en 1995. L'eau utilisée pour l'irrigation est issue de deux puits profonds dont l'eau est saumâtre. Cette eau saumâtre est utilisée dans les périmètres drainés, mais est aussi revendue illégalement en dehors de ces derniers, ce qui a des conséquences non négligeables sur la qualité des sols. Les sècheresses fréquentes et l'installation des périmètres drainés ont conduit au progressif abandon des pratiques agricoles anciennes. 
4 Il apparait donc clairement que l'extension spatiale de la salinité est associée à des phénomènes naturels (extension spatiale des sebkhas) qui sont renforcés par des pratiques anthropiques (abandon des pratiques anciennes et mauvaise gestion des eaux d'irrigation).

5 Les deux phénomènes (naturel et anthropique) ne touchent cependant pas les mêmes espaces. D'une part, l'abandon des pratiques culturales anciennes a pu accélérer l'extension de la salinité sur les bordures des sebkhas. Une de ces pratiques, le kabour, consistait à lessiver, amender et cultiver les bordures de sebkhas lors des années pluvieuses (Fehri, 2011), ce qui revient à diminuer ponctuellement les taux de salinité sur les marges des sebkhas et à freiner ou du moins perturber leur extension. D'autre part, l'irrigation des parcelles avec de l'eau saumâtre touche aujourd'hui les espaces agricoles relativement éloignés des sebkhas. Ces espaces sont donc nouvellement salinisés par une nouvelle pratique anthropique.

6 Ainsi, deux types d'espaces semblent être vulnérables à la salinisation des sols : les bordures de sebkhas et les parcelles agricoles irriguées ne bénéficiant pas du réseau de drainage. Pour vérifier cette hypothèse, j'ai tenté de comprendre la répartition spatiale de la salinité. Cette dernière a été appréhendée grâce, entre autres, à un transect réalisé depuis le cœur du périmètre irrigué et drainé de Melitta vers le sud et la mer en traversant une zone agricole dont les parcelles sont souvent irriguées, mais non drainées, puis un espace de palmeraie abandonnée et enfin la sebkha Essendouk (figure 2). Les échantillons de sols pour l'étude de la salinité, réalisés à $30 \mathrm{~cm}$ de profondeur, ont été prélevés en fonction du paysage rencontré afin d'établir une corrélation fine entre le paysage, les pratiques agricoles et la salinité. Des espaces plus ou moins vulnérables et touchés par la salinité ont été traversés. Ceux dont la salinité est la moins élevée sont situés sur les espaces "hauts ", non irrigués et dans le périmètre drainé (figure 2 - points 1, 4 et 5). Les parcelles irriguées, mais non drainées présentent quant à elles une salinité deux fois plus importante que les précédentes (figure 2point 2). Certaines parcelles sont d'ailleurs laissées à l'abandon du fait d'une salinité plus importante qu'alentour qui est associée à l'irrigation, mais aussi à la topographie ( figure 2 - point 3). La zone de transition entre espaces agricoles et sebkhas présente des taux de sel plus importants (figure 2 - point 6 et 7). Sur ces espaces que nous appelons les bordures de sebkhas, les palmiers meurent en même temps que la salinité augmente. Enfin dans la sebkha, la végétation est basse et halophile et les sols très salés (figure 2 point $8,9,10$ et 11). La corrélation entre type de paysage et taux de salinité relatif est donc conforme à notre hypothèse. 
Figure 2. Succession végétale des sebkhas aux palmeraies et détection de la bordure de sebkha : exemple de la sebkha Essendouk au sud de Gharbi

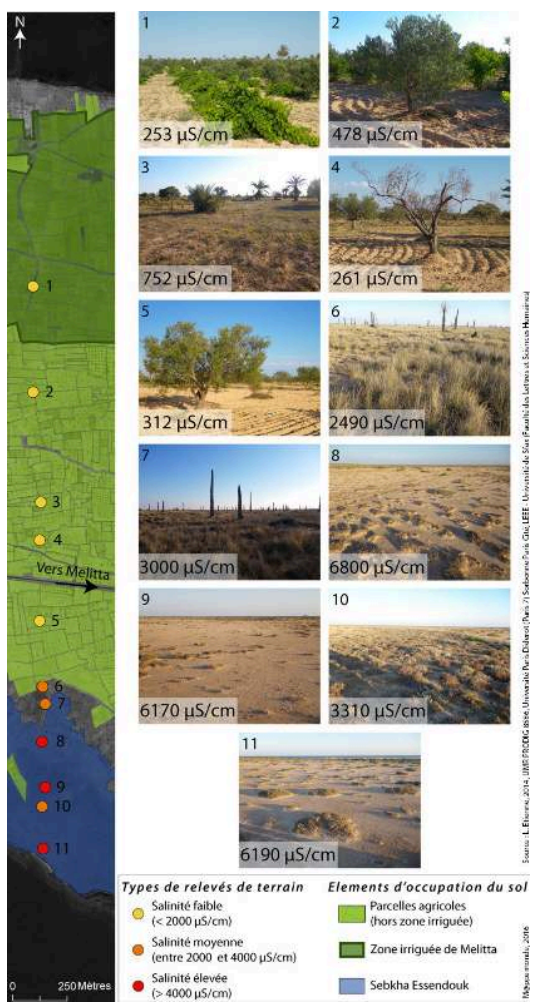

1. Parcelle agricole irriguée dans le périmètre autorisé bénéficiant d'un réseau de drainage. 2. Parcelle agricole irriguée hors du périmètre autorisé ne bénéficiant pas du réseau de drainage. 3 . Parcelle agricole abandonnée, végétation basse spontanée. 4. Parcelle agricole non irriguée. Notez les pratiques culturales différentes entre zones irriguées (création d'un bourrelet de terre à environ 20-30 $\mathrm{cm}$ autour du tronc de chaque arbre) et zone d'agriculture en sec (tronc couvert de terre sur $15-20 \mathrm{~cm}$ de haut). 5. Parcelle agricole non irriguée. 6. Zone de palmeraie dominée par la présence d'Alpha, les palmiers sont vivants. 7. Zone de palmeraie en déclin, l'Alpha domine et les palmiers sont morts. 8. Zone de sebkhas végétalisée, végétation halophile dispersée (non jointe) en petite boules. 9. Végétation halophile éparse. 10. Végétation halophile jointe et plus haute qu'en 8 et 9 . 11. Même cas qu'en 8.

Sources : Image SPOT 5 de 2010 ; photographies et relevés de salinités réalisés en juin 2011

7 Dans ma thèse j'ai pu montrer que les surfaces menacées par à la salinisation se sont étendues depuis les années 1960. Deux types d'espaces y sont ainsi vulnérables: les bordures des sebkhas et les espaces irrigués, mais non drainés. Pour mieux comprendre le paradoxe de l'irrigation avec de l'eau saumâtre des sols non drainés, il est nécessaire de changer d'échelle et de prendre en compte l'évolution de l'ensemble du socio-écosystème de l'archipel dans toute sa dimension physique, économique et politique (Étienne, 2014). 


\section{BIBLIOGRAPHIE}

DAHECH S., BELTRANDO G. (2012). « Observed temperature evolution in the City of Sfax (Middle Eastern Tunisia) for the period 1950-2007 ». Climatic Change, vol. 114, n 3-4, p. 689-706.

FEHRI N. (2011). « La palmeraie des îles Kerkennah (Tunisie), un paysage d'oasis maritime en dégradation : déterminisme naturel ou responsabilité anthropique? ». Physio-Géo, vol. 5, p. $167-189$.

ARNOULD P., HOTYAT M. (2003). Eau et environnement : Tunisie et milieux méditerranéens. Lyon : ENS Éditions, $212 \mathrm{p}$.

SAIDANI N. (2007). Rapport du centre hydrographique et océanographique de la marine nationale de la Tunisie. 5 p. En ligne : https://www.gloss-sealevel.org/publications/documents/ tunisia_gex2007.pdf

INDEX

Mots-clés : prix de thèse 2015

\section{AUTEUR}

\section{LUCILE ÉTIENNE}

Université Paris Diderot 